\title{
Avaliação de Herbicidas para Dois Cultivares de Mandioca ${ }^{1}$
}

\author{
Selectivity of Herbicide Alternatives for Two Cassava Cultivars
}

\author{
BIFFE, D.F..$^{2,7}$, CONSTANTIN, J.3,8, OLIVEIRA JR., R.S. ${ }^{3,8}$, RIOS, F.A. ${ }^{4,8}$, FRANCHINI, L.H.M. ${ }^{4,8}$, \\ GEMELLI, A. ${ }^{6,8}$, ARANTES, J.G.Z. ${ }^{5,8}$, RAIMONDI, M.A. ${ }^{5,8}$ e BLAINSKI, E. ${ }^{4,8}$
}

\begin{abstract}
RESUMO - É importante avaliar a tolerância de variedade de mandioca a novas alternativas de controle químico, com o intuito de ampliar as opções disponíveis. Este trabalho teve como objetivo avaliar a seletividade de herbicidas aplicados em pré-emergência, para duas importantes variedades de mandioca cultivadas no Estado do Paraná. Os herbicidas e respectivas doses (g i.a. ha-1) avaliadas foram: diuron (400 e 800), metribuzin (360 e 720), isoxaflutole (60), atrazine (720), S-metolachlor (1.920) e as misturas ametryn + clomazone $(1.350+1.900)$, ametryn+trifluralin $(1.500+1.350)$, isoxaflutole + metribuzin $(60+320)$, isoxaflutole+diuron $(60+400)$, combinados com uso de uma testemunha dupla adjacente a cada tratamento. Os cultivares utilizados neste trabalho foram Fécula Branca e Fibra. Apenas o herbicida S-metolachlor, para ambos os cultivares, e metribuzin (360 g i.a. ha ${ }^{-1}$ ), para o cultivar Fibra, não provocaram injúrias. Atrazine provocou redução de estande para o cultivar Fécula Branca aos 60 DAP, mas não foi detectada redução na altura de plantas. Tanto atrazine (para os dois cultivares) quanto diuron na dose de $800 \mathrm{~g}$ i.a. ha-1 (para o cultivar Fécula Branca) afetaram a produtividade de raizes. Dessa forma, atrazine foi considerado não seletivo para ambos os cultivares, e a maior dose de diuron foi também considerada não seletiva para o cultivar Fécula Branca. Há diferenças de tolerância entre os cultivares, sendo o Fibra, de modo geral, mais tolerante aos herbicidas avaliados.
\end{abstract}

Palavras-chave: variedades, diuron, isoxaflutole, metribuzin, atrazine, S-metolachlor.

\begin{abstract}
It is important to evaluate the tolerance of cassava varieties under new weed chemical control alternatives. Thus, this study aimed to evaluate the selectivity of herbicides, applied at preemergence, fortwo important cassava varieties grown in the state of Paraná, Brazil. The herbicides and respective doses ( $g$ a.i. ha $\mathrm{a}^{-1}$ ) were: diuron (400 and 800), metribuzin (360 and 720), isoxaflutole (60), atrazine (720), S-metolachlor (1,920) and mixtures ametryn+clomazone (1,350+1,900), ametryn + trifluralin (1,500+1,350), isoxaflutole + metribuzin (60+320), isoxaflutole + diuron (60+400), combined with the use of a dual checkl adjacent to each treatment. The cultivars used were Fécula Branca and Fibra. Only herbicide S-metolachlor applied on both cultivars and metribuzin (360 g a.e. ha $\mathrm{h}^{-1}$ ) applied on Fibra did not cause any injury. Atrazine reduced plant density only for Fécula Branca at 60 DAS (days after seeding) but no difference in plant height was detected. Atrazine reduced plant density for cultivar Fécula Branca at 60 DAS, but no plant height reduction was detected. Both atrazine (for both cultivars) and diuron at a dose of $800 \mathrm{~g}$ a.i. ha ${ }^{-1}$ (for Fécula Branca) affected root productivity. Thus, atrazine was considered non-selective for both cultivars and the highest diuron dose was also considered non-selective for the cultivar Fécula branca. Differences in tolerance were verified among the cultivars used in this experiment, with Fibra in general being more tolerant to the herbicides evaluated.
\end{abstract}

Keywords: varieties, diuron, isoxaflutole, metribuzin, atrazine, S-metolachlor.

1 Recebido para publicação em 25.10.2009 e na forma revisada em 12.11.2010.

Parte da Dissertação de Mestrado apresentada pelo primeiro autor para obtenção do grau de Mestre em Agronomia, área de concentração em Proteção de Plantas.

2 Engo-Agr ${ }^{0}$., Doutorando, Universidade Estadual de Maringá - UEM, <biffeagro@hotmail.com>; ${ }^{3}$ Professor Associado, Núcleo de Estudos Avançados em Ciência das Plantas Daninhas - NAPD/UEM, Dep. de Agronomia, Av. Colombo 5790, 87020-9000 Maringá-PR, <rsojunior@uem.br>; ${ }^{4}$ Eng-o-Agr ${ }^{\circ}$., Mestrando, UEM; ${ }^{5}$ Engo-Agr ${ }^{\circ}$., Doutorando, UEM; ${ }^{6}$ Graduando, UEM; ${ }^{7}$ Bolsista Capes; ${ }^{8}$ Bolsista CNPq.

Planta Daninha, Viçosa-MG, v. 28, n. 4, p. 807-816, 2010 


\section{INTRODUÇÃO}

A cultura da mandioca apresenta lento crescimento inicial e pequena capacidade de sombreamento, o que retarda a cobertura eficiente do solo e proporciona a germinação e emergência de sementes de plantas daninhas durante boa parte do ciclo (Lorenzi \& Dias, 1993). Outro fator que influencia o manejo de plantas daninhas é o espaçamento de plantio; quando o plantio é mais adensado, a cobertura do solo é mais precoce, minimizando a emergência das infestantes. Esses fatores, juntos, proporcionam baixa capacidade competitiva com a comunidade infestante e oneram o custo de produção da mandioca. Isso é facilmente constatado pela participação da mão de obra destinada às capinas manuais e mecânicas para o controle das plantas infestantes, que, segundo Peressin (1998), é da ordem de 30 a $45 \%$ do custo total de produção da cultura.

As perdas de produção na cultura da mandioca, causadas pelas plantas infestantes, são bastante variáveis em função, principalmente, da diversidade de situações nas quais a cultura pode ser instalada (época de plantio muito extensa e variada de região para região) ou conduzida (por um ciclo vegetativo, por dois ciclos vegetativos e, mais raramente, em determinadas situações, dependendo da variedade, por até três ciclos vegetativos) (Peressin, 1998).

A capina com enxada, devido aos inconvenientes de alto custo e baixo rendimento (15 a 20 homens dia $^{-1} \mathrm{ha}^{-1}$ ), torna-se impraticável em grandes lavouras (Lorenzi \& Peressin, 1989; Santos \& Paiva, 2002). Dessa forma, uma importante alternativa que pode ser tomada é o uso de herbicidas.

Entre as características que deve apresentar um herbicida, o grau de seletividade à cultura é talvez a mais importante do ponto de vista agronômico, pois de nada adianta o produto promover excelente controle das plantas daninhas, mas causar perdas de produtividade na planta cultivada (Pereira et al., 2001).

Conforme Velini et al. (1992), a seletividade é a capacidade de um determinado herbicida eliminar plantas daninhas que se encontram no mesmo ambiente da cultura sem causar efeitos fitotóxicos capazes de reduzir a produtividade e/ou prejudicar a qualidade do produto colhido. A seletividade é, portanto, detectada por meio de avaliações de fitointoxicação e do rendimento da cultura.

Melhorança (1999) relata exemplos de herbicidas que podem reduzir a produtividade das culturas sem causar efeitos visualmente detectáveis de fitointoxicação e de outros que podem provocar injúrias tóxicas evidentes na cultura e não causar redução na produtividade.

Oliveira Jr. et al. (2001a) conduziram trabalho que teve como objetivo analisar a eficácia e a seletividade de diversos herbicidas aplicados em área de plantio direto de mandioca no município de Araruna-PR, utilizando o cultivar Espeto. Considerando tanto a seletividade quanto a eficácia, os melhores resultados foram obtidos com as misturas [ametryn+clomazone] $\left[1,50+1,00 \mathrm{~kg} \mathrm{ha}^{-1}\right]$ e ametryn + diuron $(0,62+$ $0,96 \mathrm{~kg} \mathrm{ha}^{-1}$ ), ambas aplicadas em pós-emergência das plantas daninhas e quando a mandioca estava com 5 a $10 \%$ das brotações emergidas. Metolachlor+metribuzin $\left(1,68+0,24 \mathrm{~kg} \mathrm{ha}^{-1}\right) \mathrm{e}$ metribuzin $\left(0,48 \mathrm{~kg} \mathrm{ha}^{-1}\right)$, aplicados em préemergência, e ametryn $\left(1,50 \mathrm{~kg} \mathrm{ha}^{-1}\right)$, em pós-emergência, proporcionaram excelente controle das plantas daninhas presentes na área do experimento, porém reduziram significativamente a produção de raízes. Ametryn, oxyfluorfen e sulfentrazone, aplicados em préemergência, não foram seletivos à cultura da mandioca, sendo, portanto, desaconselhável seu uso. Acetolachlor, alachlor, flumioxazin, metolachlor e trifluralin, todos aplicados em pré-emergência, e flumiclorac-pentil, em pósemergência, apesar do baixo nivel de controle, foram considerados seletivos à cultura da mandioca.

A seletividade de um herbicida, em muitos casos, não pode ser atribuída exclusivamente à espécie cultivada, mas também ao cultivar utilizado. O comportamento diferenciado das cultivares frente à aplicação dos herbicidas tem sido ressaltado em resultados de pesquisa e em observações em campo (Pereira et al., 2001).

Diversos trabalhos evidenciaram diferenças intervarietais de sensibilidade a herbicidas em várias culturas. Para a cultura do milho, por exemplo, Damião Filho et al. (1996) compararam o comportamento de nove híbridos 
de milho em relação à sensibilidade ao nicosulfuron, concluindo que em pelo menos um deles houve redução da produtividade. Land et al. (1999) demonstraram que o indice de resistência de plantas de milho ao trifluralin é uma característica genética e que descendentes oriundos do cruzamento entre dois híbridos resistentes têm tolerância ao herbicida ainda maior que suas progênies.

Wychen et al. (1999) avaliaram a tolerância de híbridos de milho-doce ao isoxaflutole, concluindo que, além da variabilidade de suscetibilidade inerente aos híbridos, a tolerância variava em função do tipo de solo em que a semeadura era feita. Cavalieri et al. (2008), avaliando a seletividade do herbicida nicosulfuron a cinco híbridos de milho, detectaram que um deles apresentou redução significativa de produção.

Wilson (1999) avaliou nove cultivares de beterraba utilizando herbicidas aplicados em pós-emergência e mostrou que a tolerância variava entre cultivares e até mesmo entre anos de plantio, dentro do mesmo cultivar.

Pereira et al. (2001) detectaram diferenças significativas quanto à sensibilidade inicial de alguns cultivares de cana-de-açúcar (Saccharum officinarum) aos diversos herbicidas aplicados em pré-emergência.

Especificamente em relação à mandioca, Oliveira Jr. et al. (2001b), em trabalho realizado em Araruna-PR com cinco diferentes cultivares de mandioca (Espeto, Mico, Fécula Branca, IAC-14 e Fibra), detectaram que o tratamento [ametryn+clomazone] [1.350+ $900 \mathrm{~g}$ i a ha-1] não foi seletivo para o cultivar IAC-14, afetando sua produtividade, enquanto para os demais cultivares o tratamento não provocou decréscimo na produção, evidenciando assim diferenças de seletividade.

Devido à carência de produtos registrados para a cultura da mandioca e ao número limitado de estudos que avaliem a seletividade de novos tratamentos herbicidas e possiveis diferenças entre cultivares, objetivou-se neste trabalho avaliar a seletividade de herbicidas potenciais para uso na cultura da mandioca, assim como possiveis diferenças de seletividade entre os cultivares, visando atender à necessidade dos mandiocultores da região noroeste do Estado do Paraná.

\section{MATERIAL E MÉTODOS}

O trabalho foi desenvolvido em área pertencente à Agroindustrial Amidos Pasquini, no município de Nova Esperança, PR, a 23॰06'14" de latitude sul, 52 ' 12'04" de longitude oeste de Greenwich e a $530 \mathrm{~m}$ de altitude. Segundo a classificação de Köppen, o clima para a região é do tipo Cfa, mesotérmico úmido, com chuvas de verão e de outono e verão quente.

O solo da área experimental foi identificado como Latossolo Vermelho distrófico (Embrapa, 1999) de textura arenosa. As análises químicas e granulométricas encontramse na Tabela 1, e a precipitação acumulada mensalmente durante a condução do experimento, na Tabela 2.

O preparo do solo adotado foi o convencional, realizando-se a descompactação do solo com um subsolador de haste modelo Ikeda, a uma profundidade aproximada de $0,40 \mathrm{~m}$. Posteriormente, realizaram-se duas gradagens,

Tabela 1 - Resultado das análises químicas e granulométricas do solo utilizado no experimento de campo (camada de 0 a $20 \mathrm{~cm}$ de profundidade). Nova Esperança-PR, 2007

\begin{tabular}{|c|c|c|c|c|c|c|c|c|c|c|}
\hline \multicolumn{2}{|c|}{$\mathrm{pH}$} & $\mathrm{Al}^{3+}$ & $\mathrm{H}^{+}+\mathrm{Al}^{3+}$ & $\mathrm{Ca}^{2+}$ & $\mathrm{Mg}^{2}$ & $\mathrm{~K}^{+}$ & SB & CTC & \multirow{2}{*}{$\begin{array}{c}\mathrm{P} \\
\left(\mathrm{mg} \mathrm{dm}^{-3}\right)\end{array}$} & \multirow{2}{*}{$\frac{\mathrm{C}}{\left(\mathrm{g} \mathrm{dm}^{-3}\right)}$} \\
\hline$\left(\mathrm{CaCl}_{2}\right)$ & $\left(\mathrm{H}_{2} \mathrm{O}\right)$ & \multicolumn{7}{|c|}{$\left(\mathrm{cmol}_{\mathrm{c}} \mathrm{dm}^{-3}\right)$} & & \\
\hline 5,2 & 6,1 & 0 & 2,54 & 1,05 & 0,46 & 0,05 & 1,56 & 4,1 & 2,5 & 3,16 \\
\hline \multicolumn{3}{|c|}{ Areia grossa } & \multicolumn{3}{|c|}{ Areia fina } & \multicolumn{3}{|c|}{ Silte } & \multicolumn{2}{|c|}{ Argila } \\
\hline \multicolumn{11}{|c|}{$\left(\mathrm{g} \mathrm{kg}^{-1}\right)$} \\
\hline \multicolumn{3}{|c|}{310} & \multicolumn{3}{|c|}{530} & \multicolumn{3}{|c|}{20} & \multicolumn{2}{|c|}{140} \\
\hline
\end{tabular}

Fonte: Laboratório de Solos da Universidade Estadual de Maringá. 
Tabela 2 - Precipitação pluvial acumulada mensalmente durante a condução do experimento. Nova Esperança-PR

\begin{tabular}{|c|c|c|c|c|c|c|c|c|c|c|c|c|c|c|c|}
\hline \multicolumn{16}{|c|}{ Precipitação mensal (mm) } \\
\hline \multicolumn{2}{|c|}{2006} & \multicolumn{12}{|c|}{2007} & \multicolumn{2}{|c|}{2008} \\
\hline nov & dez & jan & fev & mar & $\mathrm{abr}$ & maio & jun & jul & ago & set & out & nov & dez & jan & fev \\
\hline 132 & 270 & 420 & 273 & 197 & 119 & 38 & 0 & 154 & 15 & 5 & 125 & 184 & 159 & 92 & 83 \\
\hline
\end{tabular}

Fonte: Cocamar Nova Esperança.

primeiramente com grade aradora a $0,20 \mathrm{~m}$ de profundidade e depois com o uso da grade niveladora, com a finalidade de destorroamento e homogeneização do terreno.

Para caracterizar as possiveis diferenças de seletividade entre cultivares de mandioca, foram conduzidos dois experimentos simultaneamente: um utilizando o cultivar Fécula Branca e outro com o cultivar Fibra. Para ambos os experimentos, a mesma metodologia a seguir foi utilizada.

O plantio ocorreu dia 1ㅇ de novembro de 2006, utilizando-se uma plantadeira do modelo Bazuca II (Planticenter) com duas linhas de plantio. As manivas foram distribuídas a aproximadamente $0,1 \mathrm{~m}$ de profundidade, em um espaçamento de 0,6 m entre manivas na linha de plantio e $0,9 \mathrm{~m}$ entre linhas. No sulco de plantio foi feita a aplicação de $200 \mathrm{~kg} \mathrm{ha}^{-1}$ do fertilizante formulado 04-14-08.

Cada unidade experimental foi constituída por seis linhas de $5 \mathrm{~m}$ de comprimento, perfazendo uma área de $30 \mathrm{~m}^{2}$, sendo, porém, consideradas como área útil apenas as quatro linhas centrais, descontado $0,5 \mathrm{~m}$ de cada extremidade $\left(14,4 \mathrm{~m}^{2}\right)$.

Intercalada a cada parcela dos tratamentos com herbicida, foi utilizada uma testemunha dupla adjacente. O sistema de testemunhas duplas adjacentes foi anteriormente descrito por Fagliari et al. (2001) e Meschede et al. (2004) e consiste no aumento do número de testemunhas dentro de cada repetição, o que corresponde a uma parcela não tratada ao lado de cada parcela que recebeu o tratamento.

A técnica de testemunhas duplas foi inicialmente proposta para avaliação da seletividade de herbicidas em cana-de-açúcar. Mais recentemente, foi utilizada também no estudo da seletividade de herbicidas na cultura da soja (Meschede et al., 2004;
Jaremtchuk et al., 2008). De modo geral, utiliza-se a média das testemunhas adjacentes como referência das características avaliadas para cada parcela-tratamento. Esse tipo de experimento confere maior controle sobre a variabilidade do meio, sobretudo quando se utiliza o tradicional delineamento em blocos casualizados, com uma única testemunha por bloco (Meschede et al., 2004).

As aplicações dos tratamentos foram realizadas em duas épocas. Um dia após o plantio, realizou-se a aplicação dos herbicidas em préemergência (PRÉ); o tratamento [ametryn+ clomazone] foi aplicado 10 dias após o plantio (DAP), em pós-emergência inicial (PÓSi), quando 5 a $10 \%$ das plantas de mandioca já haviam emergido. Os tratamentos herbicidas encontram-se na Tabela 3.

Os herbicidas foram aplicados usando-se um pulverizador costal pressurizado com $\mathrm{CO}_{2}$, provido de barra com quatro pontas de jato leque, $\mathrm{AD} 110.02$, espaçadas entre si de $0,5 \mathrm{~m}$, com volume de calda de $200 \mathrm{~L} \mathrm{ha}^{-1}$ e pressão de aspersão de 3,0 $\mathrm{kgf} \mathrm{cm}^{-2}$. Em todas as parcelas o controle de plantas daninhas foi realizado por meio de capinas, a fim de evitar perdas de produtividade por interferência delas.

Foram realizadas avaliações de fitointoxicação da cultura aos 30, 45 e 60 dias após o plantio (DAP), por meio da escala elaborada pela European Weed Research Council (1964), que segue uma escala de 1 a 9 , em que 1 corresponde à ausência de sintomas e 9 à morte completa das plantas.

O estande de cada tratamento foi obtido em duas avaliações (30 e 60 DAP), e em ambas foi realizada contagem das plantas emergidas em três metros, nas duas linhas centrais da parcela. Para avaliação de altura, procedeu-se à avaliação de 10 plantas nas duas linhas centrais de cada parcela, aos 60 DAP. A medição da altura foi feita do colo da planta até a 
Tabela 3 - Tratamentos utilizados no experimento. Nova Esperança - Paraná

\begin{tabular}{|c|c|c|c|}
\hline $\begin{array}{c}\text { Trata- } \\
\text { mento }\end{array}$ & Herbicida & Modalidade & $\begin{array}{c}\text { Dose } \\
\left(\mathrm{g} \text { i.a. } \mathrm{ha}^{-1}\right)\end{array}$ \\
\hline I & [Ametryn + Clomazone] & PÓSi & $1.350+900$ \\
\hline II & Ametryn + Trifluralin & PRÉ & $1.500+1.350$ \\
\hline III & Isoxaflutole & PRÉ & 60 \\
\hline IV & Metribuzin & PRÉ & 360 \\
\hline V & Diuron & PRÉ & 400 \\
\hline VI & Isoxaflutole + Metribuzin & PRÉ & $60+360$ \\
\hline VII & Isoxaflutole + Diuron & PRÉ & $60+400$ \\
\hline VIII & Metribuzin & PRÉ & 720 \\
\hline IX & Diuron & PRÉ & 800 \\
\hline X & Atrazine & PRÉ & 1.500 \\
\hline XI & S-metolachlor & PRÉ & 1.920 \\
\hline
\end{tabular}

PÓSi: pós-emergência inicial; PRÉ: pré-emergência.

inserção da última folha expandida na haste principal.

A colheita ocorreu 14 meses após o plantio e, para determinação da produtividade, foram colhidas manualmente as plantas presentes nas quatro linhas centrais, deixando como bordadura $0,5 \mathrm{~m}$ de cada parcela. As raízes foram separadas e pesadas em balança, imediatamente após a colheita.

Para ambos os experimentos o delineamento experimental utilizado foi o de blocos ao acaso, em parcelas subdivididas, com quatro repetições. Quanto aos dados de fitointoxicação, foram obtidos apenas as médias, e para as demais avaliações os dados foram analisados comparando-se os tratamentos com a média das testemunhas duplas adjacentes. Neste caso, os dados foram submetidos à análise de variância pelo teste $\mathrm{F}$, e as médias, comparadas pelo teste de Tukey a $5 \%$ de probabilidade, utilizando o pacote estatístico SAEG 5.0.

\section{RESULTADOS E DISCUSSÃO}

O herbicida [ametryn+clomazone] apresentou as maiores notas de injúria aos 30 DAP (Tabela 4); os sintomas mais evidentes consistiam em amarelecimento generalizado e necrose das folhas. No entanto, a partir de 60 DAP as plantas se recuperaram e as folhas novas não apresentaram mais sintomas de fintoxicação. Resultados semelhantes foram obtidos por Oliveira Jr. et al. (2001b), em que as injúrias mais severas aconteceram aos 37 DAP; a partir dessa data, a cultura se restabeleceu e os sintomas de injúrias não eram mais visiveis.

Para o herbicida atrazine na avaliação de 30 DAP não havia sintomas visiveis de injúrias; contudo, aos 45 DAP as plantas passaram a apresentar necrose generalizada, ocorrendo morte de algumas plantas nas parcelas até os 60 DAP (Tabela 4).

Os tratamentos com ametryn+trifluralin, isoxaflutole, diuron, isoxaflutole+metribuzin, isoxaflutole+diuron, metribuzin (720 g i.a. ha $\left.{ }^{-1}\right)$ e diuron (800 g i.a. ha-1), em ambos os cultivares, apresentaram sintomas leves a moderados de fitointoxicação, sendo caracterizados por amarelecimento foliar, porém sem evolução

Tabela 4 - Fitointoxicação aos 30, 45 e 60 DAP para os cultivares Fécula Branca e Fibra

\begin{tabular}{|l|c|c|c|c|c|c|c|}
\hline \multirow{2}{*}{ Tratamento } & \multirow{2}{*}{$\begin{array}{c}\text { Dose } \\
\left.\text { (g i.a. ha }{ }^{-1}\right)\end{array}$} & \multicolumn{5}{|c|}{ Fitointoxicacão (EWRC) } \\
\cline { 3 - 8 } & & F. Branca (DAP) & \multicolumn{3}{|c|}{ Fibra (DAP) } \\
\hline $\begin{array}{l}\text { [Ametryn + } \\
\text { Clomazone] }\end{array}$ & {$[1.350+900]$} & 6 & 5 & 1 & 5 & 4 & 1 \\
\hline $\begin{array}{l}\text { Ametryn + } \\
\text { Trifluralin }\end{array}$ & $1.500+1.350$ & 1 & 2 & 1 & 1 & 3 & 2 \\
\hline Isoxaflutole & 60 & 3 & 3 & 1 & 2 & 2 & 1 \\
\hline Metribuzin & 360 & 2 & 3 & 1 & 1 & 1 & 1 \\
\hline Diuron & 400 & 2 & 3 & 1 & 2 & 1 & 1 \\
\hline $\begin{array}{l}\text { Isoxaflutole }+ \\
\text { Metribuzin }\end{array}$ & $60+360$ & 2 & 5 & 1 & 2 & 4 & 1 \\
\hline $\begin{array}{l}\text { Isoxaflutole }+ \\
\text { Diuron }\end{array}$ & $60+400$ & 3 & 2 & 1 & 2 & 2 & 1 \\
\hline Metribuzin & 720 & 3 & 4 & 1 & 1 & 4 & 1 \\
\hline Diuron & 800 & 3 & 4 & 1 & 2 & 3 & 1 \\
\hline Atrazine & 1.500 & 1 & 7 & 5 & 1 & 6 & 5 \\
\hline S-metolachlor & 1.920 & 1 & 1 & 1 & 1 & 1 & 1 \\
\hline
\end{tabular}

Escala EWRC, em que 1= ausência de sintomas, 9= morte total de plantas.

Planta Daninha, Viçosa-MG, v. 28, n. 4, p. 807-816, 2010 
para necrose. Apesar de apresentarem sintomas de fitointoxicação já aos 30 DAP, as maiores injúrias foram observadas aos $45 \mathrm{DAP}$, e a partir de 60 DAP nenhum sintoma foi observado. Metribuzin na menor dose (360 g i.a. ha ${ }^{-1}$ ) promoveu sintomas leves de intoxicação aos 30 e 45 DAP apenas para o cultivar Fécula Branca, porém os sintomas desapareceram aos 60 DAP. O herbicida S-metolachlor foi o único que não causou sinais de fitointoxicação em todas as avaliações para ambos os cultivares avaliados.

De maneira geral, os sintomas mais visíveis de intoxicação ocorreram aos $45 \mathrm{DAP}$ em ambas os cultivares e de maneira mais acentuada no Fécula Branca, evidenciando haver diferença quanto à tolerância entre os dois cultivares estudados.

Aos 30 DAP não foram constatados efeitos significativos dos tratamentos com relação às respectivas testemunhas sem aplicação em ambos os cultivares. No entanto, aos 60 DAP, para o cultivar Fécula Branca, o tratamento com atrazine reduziu significativamente a população de plantas (Tabelas 6 e 7).

Esses resultados concordam com os observados por Oliveira Jr. et al. (2001b) a respeito do metribuzin (720 g i.a. ha $\left.{ }^{-1}\right)$ e das misturas [ametryn+clomazone] e ametryn+trifluralin, para os cultivares Fécula Branca e Fibra, uma vez que esses tratamentos não provocaram redução do número de plantas. Por outro lado, esses resultados contrastam com aqueles obtidos por Alabi et al. (2004), que, trabalhando com uma dose de atrazine semelhante à utilizada neste experimento (1.200 g i.a. ha $\left.{ }^{-1}\right)$, não detectaram diferenças significativas quanto ao estande em relação à testemunha.

Nenhum tratamento químico diferiu da sua respectiva testemunha no que se refere

Tabela 5 - Estande do cultivar Fécula Branca aos 30 e 60 DAP

\begin{tabular}{|c|c|c|c|c|c|c|c|}
\hline \multirow{3}{*}{ Tratamento } & \multirow{3}{*}{ Dose (g i.a. ha $\left.{ }^{-1}\right)$} & \multicolumn{6}{|c|}{ Estande (plantas ha $\left.{ }^{-1}\right)$} \\
\hline & & \multicolumn{3}{|c|}{$30 \mathrm{DAP}$} & \multicolumn{3}{|c|}{60 DAP } \\
\hline & & Trat & $\mathrm{TD}$ & Dif. & Trat & TD & Dif. \\
\hline [Ametryn + Clomazone] & $1.350+900$ & $15.555 \mathrm{a}$ & $15.278 \mathrm{a}$ & 277 & $15.558 \mathrm{a}$ & $15.555 \mathrm{a}$ & 3 \\
\hline Ametryn + Trifluralin & $1.500+1.350$ & $15.833 \mathrm{a}$ & $15.000 \mathrm{a}$ & 833 & $15.552 \mathrm{a}$ & $15.277 \mathrm{a}$ & 275 \\
\hline Isoxaflutole & 60 & $15.640 \mathrm{a}$ & $14.434 \mathrm{a}$ & 1.206 & 14.998 a & $14.722 \mathrm{a}$ & 276 \\
\hline Metribuzin & 360 & $16.110 \mathrm{a}$ & $16.115 \mathrm{a}$ & -5 & 14.994 a & $15.883 \mathrm{a}$ & -889 \\
\hline Diuron & 400 & $14.166 \mathrm{a}$ & $15.006 \mathrm{a}$ & -840 & $14.167 \mathrm{a}$ & $15.558 \mathrm{a}$ & -1.391 \\
\hline Isoxaflutole + Metribuzin & $60+360$ & $15.277 \quad \mathrm{a}$ & $14.412 \mathrm{a}$ & 865 & $15.560 \mathrm{a}$ & $14.166 \mathrm{a}$ & 1.394 \\
\hline Isoxaflutole + Diuron & $60+400$ & $14.173 \mathrm{a}$ & $15.275 \mathrm{a}$ & -1.102 & 14.164 a & $15.551 \mathrm{a}$ & -1.387 \\
\hline Metribuzin & 720 & $13.889 \mathrm{a}$ & $15.559 \mathrm{a}$ & -1670 & $14.996 \mathrm{a}$ & $15.883 \mathrm{a}$ & -887 \\
\hline Diuron & 800 & $14.731 \mathrm{a}$ & $15.558 \mathrm{a}$ & -827 & $14.722 \mathrm{a}$ & $15.000 \mathrm{a}$ & -278 \\
\hline Atrazine & 1.500 & $14.718 \mathrm{a}$ & $16.111 \mathrm{a}$ & -1.393 & $12.777 \mathrm{~b}$ & $16.114 \mathrm{a}$ & -3.337 \\
\hline S-metolachlor & 1.920 & $14.725 \mathrm{a}$ & $16.108 \mathrm{a}$ & -1.383 & $14.444 \quad \mathrm{a}$ & $15.554 \mathrm{a}$ & -1.110 \\
\hline \multicolumn{2}{|l|}{$\mathrm{CV}(\%)$} & \multicolumn{3}{|c|}{7,9} & \multicolumn{3}{|c|}{7,3} \\
\hline \multicolumn{2}{|c|}{ DMS (Tukey, $5 \%$ ) } & \multicolumn{3}{|c|}{$1.719,7$} & \multicolumn{3}{|c|}{$1.578,6$} \\
\hline \multicolumn{2}{|c|}{ DMS (Tukey, $10 \%$ ) } & \multicolumn{3}{|c|}{$1.429,5$} & \multicolumn{3}{|c|}{$1.312,4$} \\
\hline
\end{tabular}

Médias seguidas na linha pela mesma letra não diferem entre si pelo teste de Tukey a $5 \%$ de probabilidade. Trat: média obtida no tratamento; TD: média obtida na testemunha dupla; Dif: diferença entre a média do tratamento e da testemunha dupla. 
Tabela 6 - Estande do cultivar Fibra aos 60 DAP

\begin{tabular}{|c|c|c|c|c|c|c|c|}
\hline \multirow{3}{*}{ Tratamento } & \multirow{3}{*}{ Dose (g i.a. ha $\left.{ }^{-1}\right)$} & \multicolumn{6}{|c|}{ Estande (plantas ha ${ }^{-1}$ ) } \\
\hline & & \multicolumn{3}{|c|}{$30 \mathrm{DAP}$} & \multicolumn{3}{|c|}{60 DAP } \\
\hline & & Trat & $\mathrm{TD}$ & Dif. & Trat & TD & Dif. \\
\hline [Ametryn + Clomazone] & $1.350+900$ & $16.388 \quad \mathrm{a}$ & $16.118 \quad \mathrm{a}$ & 270 & $15.560 \quad \mathrm{a}$ & $14.456 \mathrm{a}$ & 1.104 \\
\hline Ametryn + Trifluralin & $1.500+1.350$ & $15.557 \quad \mathrm{a}$ & $15.546 \mathrm{a}$ & 11 & $14.730 \quad \mathrm{a}$ & $14.434 \mathrm{a}$ & 296 \\
\hline Isoxaflutole & 60 & $15.556 \mathrm{a}$ & $15.886 \mathrm{a}$ & -330 & $14.729 \quad \mathrm{a}$ & $15.008 \mathrm{a}$ & -279 \\
\hline Metribuzin & 360 & $15.227 \quad \mathrm{a}$ & $16.114 \mathrm{a}$ & -887 & $14.167 \quad \mathrm{a}$ & $15.547 \quad \mathrm{a}$ & -1.380 \\
\hline Diuron & 400 & $15.883 \mathrm{a}$ & $16.648 \mathrm{a}$ & -765 & $15.279 \quad \mathrm{a}$ & $15.564 \mathrm{a}$ & -285 \\
\hline Isoxaflutole + Metribuzin & $60+360$ & $16.111 \mathrm{a}$ & $16.120 \mathrm{a}$ & -9 & $15.555 \mathrm{a}$ & $15.833 \mathrm{a}$ & -278 \\
\hline Isoxaflutole + Diuron & $60+400$ & $15.889 \quad \mathrm{a}$ & $16.117 \quad \mathrm{a}$ & -228 & $14.734 \quad \mathrm{a}$ & $15.561 \quad \mathrm{a}$ & -827 \\
\hline Metribuzin & 720 & $14.995 \mathrm{a}$ & $15.841 \quad \mathrm{a}$ & -846 & $14.434 \mathrm{a}$ & $15.548 \quad \mathrm{a}$ & -1.114 \\
\hline Diuron & 800 & $15.886 \mathrm{a}$ & $15.561 \quad \mathrm{a}$ & 325 & $15.581 \quad \mathrm{a}$ & $14.434 \mathrm{a}$ & 1.147 \\
\hline Atrazine & 1.500 & $16.385 \mathrm{a}$ & $15.558 \mathrm{a}$ & 827 & $12.777 \quad \mathrm{a}$ & $15.276 \mathrm{a}$ & -2.499 \\
\hline S-metolachlor & 1.920 & $14.443 \mathrm{a}$ & $14.723 \mathrm{a}$ & -280 & $13.889 \quad \mathrm{a}$ & $16.110 \mathrm{a}$ & -2.221 \\
\hline \multicolumn{2}{|l|}{$\mathrm{CV}(\%)$} & \multicolumn{3}{|c|}{8,7} & \multicolumn{3}{|c|}{10,5} \\
\hline \multicolumn{2}{|c|}{ DMS (Tukey, $5 \%$ ) } & \multicolumn{3}{|c|}{$1.976,0$} & \multicolumn{3}{|c|}{$2.271,0$} \\
\hline \multicolumn{2}{|c|}{ DMS (Tukey, $10 \%$ ) } & \multicolumn{3}{|c|}{$1.643,0$} & \multicolumn{3}{|c|}{$1.887,0$} \\
\hline
\end{tabular}

Médias seguidas na linha pela mesma letra não diferem entre si pelo teste de Tukey a $5 \%$ de probabilidade. Trat: média obtida no tratamento; TD: média obtida na testemunha dupla; Dif: diferença entre a média do tratamento e a da testemunha dupla.

à altura das plantas aos 60 DAP, para ambos os cultivares (Tabela 7). Esse fato demonstra que, mesmo sofrendo sintomas de injúrias aparentes após a aplicação dos herbicidas, a partir dos 60 DAP essas injúrias não alteraram o desenvolvimento da cultura.

Novamente, os resultados encontrados para metribuzin (360 g i.a. ha ${ }^{-1}$ ) e para as misturas [ametryn+clomazone] e ametryn+ trifluralin, para Fécula Branca e Fibra, estão de acordo com os observados anteriormente por Oliveira Jr. et al. (2001b), que não detectaram diferença desses tratamentos em relação à testemunha não aplicada aos 73 DAP.

Os resultados do rendimento de raízes do cultivar Fécula Branca encontram-se na Tabela 8, onde se observa que apenas os tratamentos com diuron (800 g i.a. ha ${ }^{-1}$ ) e atrazine ( $1.500 \mathrm{~g}$ i.a. ha $\mathrm{h}^{-1}$ ) afetaram a produção de raízes de maneira significativa em relação à testemunha, ao passo que os demais tratamentos não resultaram em perdas de produção.

Resultados semelhantes foram obtidos por Oliveira Jr. (2001b) em termos de seletividade para o metribuzin (320 g i.a. ha ${ }^{-1}$ ) e para a mistura [ametryn+clomazone], os quais não provocaram redução significativa para a produção do cultivar Fécula Branca.

Em se tratando do diuron (800 g i.a. ha-1), apesar de as plantas não mais apresentarem sintomas de intoxicação aos $60 \mathrm{DAP}$ e de não terem provocado redução significativa no estande da cultura, a produtividade foi comprometida. Portanto, a seletividade de um herbicida não deve ser baseada apenas nos sintomas de fitointoxicação, pois, de acordo com Melhorança (1999), alguns herbicidas podem reduzir a produtividade das culturas sem causar efeitos visualmente detectáveis de intoxicação, e outros podem provocar 
Tabela 7 - Altura de plantas (cm) para as cultivares Fécula Branca e Fibra aos 60 DAP

\begin{tabular}{|c|c|c|c|c|c|c|c|}
\hline \multirow{3}{*}{ Tratamento } & \multirow{3}{*}{ Dose (g i.a. ha $\left.{ }^{-1}\right)$} & \multicolumn{6}{|c|}{ Altura 60 DAP $(\mathrm{cm})$} \\
\hline & & \multicolumn{3}{|c|}{ Fécula Branca } & \multicolumn{3}{|c|}{ Fécula Branca } \\
\hline & & Trat & $\mathrm{TD}$ & Dif. & Trat & TD & Dif. \\
\hline [Ametryn + Clomazone] & $1.350+900$ & 18,8 a & 19,5 a & $-0,7$ & $20,0 \quad \mathrm{a}$ & 20,0 a & 0,0 \\
\hline Ametryn + Trifluralin & $1.500+1.350$ & 19,0 a & 19,8 a & $-0,8$ & 19,3 a & 21,8 a & $-2,5$ \\
\hline Isoxaflutole & 60 & 20,0 a & 19,0 a & 1,0 & $22,0 \quad \mathrm{a}$ & 23,5 a & $-1,5$ \\
\hline Metribuzin & 360 & 18,7 a & 19,0 a & $-0,3$ & $22,0 \quad \mathrm{a}$ & 22,5 a & $-0,5$ \\
\hline Diuron & 400 & 18,8 a & $18,0 \quad \mathrm{a}$ & 0,8 & $24,0 \quad \mathrm{a}$ & 23,8 a & 0,2 \\
\hline Isoxaflutole + Metribuzin & $60+360$ & 20,5 a & 19,8 a & 0,7 & $20,7 \quad a$ & 21,8 a & $-1,1$ \\
\hline Isoxaflutole + Diuron & $60+400$ & 20,3 a & 18,5 a & 1,8 & 24,3 a & 23,0 a & 1,3 \\
\hline Metribuzin & 720 & $21,0 \quad \mathrm{a}$ & 20,0 a & 1,0 & $22,8 \quad \mathrm{a}$ & 22,8 a & 0,0 \\
\hline Diuron & 800 & $20,0 \quad \mathrm{a}$ & 19,8 a & 0,2 & $22,3 \quad \mathrm{a}$ & 21,8 a & 0,5 \\
\hline Atrazine & 1.500 & 14,5 a & 18,8 a & $-4,3$ & 20,5 a & 24,0 a & $-3,5$ \\
\hline S-metolachlor & 1.920 & 18,3 a & 19,3 a & $-1,0$ & $21,3 \quad \mathrm{a}$ & 23,8 a & $-2,5$ \\
\hline \multicolumn{2}{|l|}{$\mathrm{CV}(\%)$} & \multicolumn{3}{|c|}{17,1} & \multicolumn{3}{|c|}{17,3} \\
\hline \multicolumn{2}{|c|}{ DMS (Tukey, $5 \%$ ) } & \multicolumn{3}{|c|}{4,8} & \multicolumn{3}{|c|}{5,5} \\
\hline \multicolumn{2}{|c|}{ DMS (Tukey, $10 \%$ ) } & \multicolumn{3}{|c|}{4,0} & \multicolumn{3}{|c|}{4,5} \\
\hline
\end{tabular}

Médias seguidas na linha pela mesma letra não diferem entre si pelo teste de Tukey a $5 \%$ de probabilidade. Trat: média obtida no tratamento; TD: média obtida na testemunha dupla; Dif: diferença entre a média do tratamento e a da testemunha dupla.

injúrias tóxicas evidentes na cultura e não causar redução na produtividade.

Atrazine (1.500 g i.a. ha $\left.{ }^{-1}\right)$ afetou significativamente o estande da cultura aos 60 DAP e a produtividade. Nesse caso, as injúrias severas detectadas na fase inicial da cultura, que levaram à morte de plantas, foram determinantes para a redução no rendimento de raízes. Esses resultados contrastam com os obtidos por Alabi et al. (2004), em trabalho realizado na Nigéria, porém cabe destacar que a dose de atrazine (1.200 g i.a. ha ${ }^{-1}$ e o cultivar utilizado por esses autores diferem dos empregados neste trabalho.

Cabe destacar a seletividade do herbicida metribuzin (360 e 720 g i.a. ha ${ }^{-1}$ ), que já era esperada para o caso da dose recomendada; no entanto, mostrou-se bastante seguro para o uso na cultura da mandioca, pois mesmo o dobro da dose recomendada não foi suficiente para redução da produção.

Quanto ao cultivar Fibra, apenas o tratamento com atrazine reduziu de maneira significativa o rendimento da cultura (Tabela 9). Novamente ficou caracterizado que alguns herbicidas, mesmo causando injúrias severas, não afetaram o rendimento da cultura. Contudo, no caso da atrazine, o alto nivel de fitointoxicação promoveu redução significativa na produtividade.

Vale destacar novamente o herbicida metribuzin, que nas duas doses avaliadas mostrou-se seletivo à cultura, e também o diuron, que, apesar de não ter registro para a cultura, em dose relativamente alta (800 g i.a. ha ${ }^{-1}$ ) não reduziu significativamente a produtividade.

Contudo, o uso de diuron na dose de 800 g i.a. ha- ${ }^{-1}$ deve ser criterioso, pois foi 
Tabela 8 - Produtividade de raízes de mandioca para o cultivar Fécula Branca

\begin{tabular}{|c|c|c|c|c|}
\hline \multirow{2}{*}{ Tratamento } & \multirow{2}{*}{$\begin{array}{c}\text { Dose } \\
\left(\mathrm{g} \text { i.a. } \mathrm{ha}^{-1}\right)\end{array}$} & \multicolumn{3}{|c|}{ Produtividade $\left(\mathrm{kg}\right.$ raízes $\left.\mathrm{ha}^{-1}\right)$} \\
\hline & & Trat & TD & Dif. \\
\hline $\begin{array}{l}{[\text { Ametryn }+} \\
\text { Clomazone }]\end{array}$ & {$[1.350+900]$} & $23.311 \mathrm{a}$ & $23.919 \mathrm{a}$ & -608 \\
\hline $\begin{array}{l}\text { Ametryn + } \\
\text { Trifluralin }\end{array}$ & $1.500+1.350$ & $22.882 \mathrm{a}$ & $23.514 \mathrm{a}$ & -631 \\
\hline Isoxaflutole & 60 & $25.946 \mathrm{a}$ & $24.504 \mathrm{a}$ & 1.442 \\
\hline Metribuzin & 360 & $17.162 \mathrm{a}$ & $16.216 \mathrm{a}$ & 946 \\
\hline Diuron & 400 & $20.743 \mathrm{a}$ & $19.865 \mathrm{a}$ & 878 \\
\hline $\begin{array}{l}\text { Isoxaflutole + } \\
\text { Metribuzin }\end{array}$ & $60+360$ & $20.811 \mathrm{a}$ & $20.304 \mathrm{a}$ & 507 \\
\hline \begin{tabular}{|l|}
$\begin{array}{l}\text { Isoxaflutole + } \\
\text { Diuron }\end{array}$ \\
\end{tabular} & $60+400$ & $21.351 \mathrm{a}$ & $21.588 \mathrm{a}$ & -236 \\
\hline Metribuzin & 720 & $21.351 \mathrm{a}$ & $20.541 \mathrm{a}$ & 811 \\
\hline Diuron & 800 & $19.257 \mathrm{~b}$ & $24.223 \mathrm{a}$ & -4.966 \\
\hline Atrazine & 1.500 & $16.014 \mathrm{~b}$ & $23.108 \mathrm{a}$ & -7.095 \\
\hline S-metolachlor & 1.920 & $20.405 \mathrm{a}$ & $21.757 \mathrm{a}$ & -1.351 \\
\hline \multicolumn{2}{|l|}{$\mathrm{CV}(\%)$} & \multicolumn{3}{|c|}{11,8} \\
\hline \multicolumn{2}{|c|}{ DMS (Tukey, 5\%) } & \multicolumn{3}{|c|}{$3.628,6$} \\
\hline \multicolumn{2}{|c|}{ DMS (Tukey, $10 \%$ ) } & \multicolumn{3}{|c|}{$3.016,3$} \\
\hline
\end{tabular}

Médias seguidas na linha pela mesma letra não diferem entre si pelo teste de Tukey a $5 \%$ de probabilidade. Trat: média obtida no tratamento; TD: média obtida na testemunha dupla; Dif: diferença entre a média do tratamento e a da testemunha dupla.

verificado que existe diferença quanto à seletividade de herbicidas entre as variedades Fécula Branca e Fibra; para a primeira, essa dose prejudicou a sua produtividade. É esperado, portanto, que haja resposta diferencial entre cultivares em relação a esse tratamento.

A diferença de seletividade de um mesmo produto em relação a diferentes cultivares já havia sido evidenciada em outras culturas por Damião Filho et al. (1996), Land et al. (1999), Wychen et al. (1999), Wilson (1999), Pereira et al. (2000) e Cavalieri et al. (2008). Para mandioca, Oliveira Jr. et al. (2001b), em trabalho realizado com cinco cultivares de mandioca (Espeto, Mico, Fécula Branca, IAC-14 e Fibra), detectaram que o tratamento [ametryn+clomazone] [1.350+900 g i.a. ha ${ }^{-1}$ ] não foi seletivo para o cultivar IAC-14, afetando sua produtividade, enquanto para os demais cultivares o tratamento não provocou decréscimo na produção, evidenciando assim a
Tabela 9 - Produtividade de raízes de mandioca obtida no cultivar Fibra

\begin{tabular}{|c|c|c|c|c|}
\hline \multirow{2}{*}{ Tratamento } & \multirow{2}{*}{$\begin{array}{c}\text { Dose } \\
\left(\mathrm{g} \text { i.a. } \mathrm{ha}^{-1}\right)\end{array}$} & \multicolumn{3}{|c|}{ Produtividade $\left(\mathrm{kg}\right.$ raízes ha $\left.{ }^{-1}\right)$} \\
\hline & & Trat & TD & Dif. \\
\hline $\begin{array}{l}\text { [Ametryn }+ \\
\text { Clomazone] }\end{array}$ & {$[1.350+900]$} & $15.608 \mathrm{a}$ & $17.871 \mathrm{a}$ & -2.263 \\
\hline $\begin{array}{l}\text { Ametryn + } \\
\text { Trifluralin }\end{array}$ & $1.500+1.350$ & $16.486 \mathrm{a}$ & $16.778 \mathrm{a}$ & -292 \\
\hline Isoxaflutole & 60 & $18.581 \mathrm{a}$ & $18.615 \mathrm{a}$ & -34 \\
\hline Metribuzin & 360 & 18.649 a & $18.378 \mathrm{a}$ & 270 \\
\hline Diuron & 400 & $18.378 \mathrm{a}$ & $18.682 \mathrm{a}$ & -304 \\
\hline $\begin{array}{l}\text { Isoxaflutole + } \\
\text { Metribuzin }\end{array}$ & $60+360$ & $19.662 \mathrm{a}$ & $19.047 \mathrm{a}$ & 615 \\
\hline $\begin{array}{l}\text { Isoxaflutole + } \\
\text { Diuron }\end{array}$ & $60+400$ & $17.432 \mathrm{a}$ & $16.250 \mathrm{a}$ & 1.182 \\
\hline Metribuzin & 720 & $16.824 \mathrm{a}$ & $17.399 \mathrm{a}$ & -574 \\
\hline Diuron & 800 & $16.824 \mathrm{a}$ & $16.081 \mathrm{a}$ & 743 \\
\hline Atrazine & 1.500 & $10.405 \mathrm{~b}$ & $20.765 \mathrm{a}$ & -10.359 \\
\hline S-metolachlor & 1.920 & $14.257 \mathrm{a}$ & $14.662 \mathrm{a}$ & -405 \\
\hline $\mathrm{CV}(\%)$ & & & 11,3 & \\
\hline DMS (T & $5 \%)$ & & $2.786,7$ & \\
\hline DMS (T) & $y, 10 \%)$ & & $2.316,4$ & \\
\hline
\end{tabular}

Médias seguidas na linha pela mesma letra não diferem entre si pelo teste de Tukey a $5 \%$ de probabilidade. Trat: média obtida no tratamento; TD: média obtida na testemunha dupla; Dif: diferença entre a média do tratamento e a da testemunha dupla.

diferença de seletividade de herbicidas entre cultivares de mandioca; novamente, neste trabalho foi comprovado que a mistura [ametryn+clomazone] não afeta a produção dos cultivares Fécula Branca e Fibra.

De maneira geral, os tratamentos [ametryn+clomazone] [1.350+900 g i.a. ha ${ }^{-1}$, ametryn+trifluralin $\left(1.500+1.350\right.$ g i.a. ha $\left.{ }^{-1}\right)$, metribuzin (360 e $720 \mathrm{~g}$ i.a. ha $\mathrm{a}^{-1}$ ), diuron (400 g i.a. ha-1), isoxaflutole (60 g i.a. ha-1), isoxaflutole+metribuzin $(60+360 \mathrm{~g}$ i.a. ha-1), isoxaflutole+diuron $\left(60+400 \mathrm{~g}\right.$ i.a. ha $\left.\mathrm{h}^{-1}\right) \mathrm{e}$ S-metolachlor (1.920 g i.a. ha-1), para os cultivares Fécula Branca e Fibra, mostraramse seletivos nas doses avaliadas, podendo ser usados como ferramentas no controle de plantas daninhas na cultura da mandioca.

O diuron (800 g i.a. ha- ${ }^{-1}$ ) foi seletivo apenas para o cultivar Fibra, seu uso para o cultivar Fécula Branca não é indicado, assim como de atrazine, em ambos os cultivares. 


\section{LITERATURA CITADA}

ALABI, B. S.; AYENI, O. A.; AGBOOLA, A. Economic assessment of manual and chemical control of thorny mimosa in cassava in Nigeria. New directions for a diverse planet: In: INTERNATIONAL CROP SCIENCE CONGRESS, 4. 2004, Brisbane. Proceedings... Brisbane: 2004

CAVALIERI, S. D. et al. Tolerância de híbridos de milho ao nicosulfuron. Planta Daninha, v. 26, n. 1, p. 203-214, 2008.

DAMIÃO FILHO, C. F.; MÔRO, F. V.; TAVEIRA, L. R. Respostas de híbridos de milho ao nicosulfuron. 1 - Aspectos biológicos e da produção. Planta Daninha, v. 14, n. 1, p. 3-13, 1996.

FAGLIARI, J. R.; OLIVEIRA JR., R. S.; CONSTANTIN, J Métodos de avaliação da seletividade de herbicidas para a cultura da cana-de-açúcar (Saccharum spp.). Acta Sci., v. 23, n. 5 , p. $1229-1234,2001$

EMPRESA BRASILEIRA DE PESQUISA AGROPECUÁRIA - EMBRAPA. Centro Nacional de Pesquisa de Solos. Sistema brasileiro de classificação de solos. Brasília: Embrapa Produção de Informação; Rio de Janeiro: Embrapa Solos, 1999. 412 p.

\section{EUROPEAN WEED RESEARCH COUNCIL - EWRC} Report of the $3^{\text {rd }}$ and $4 \mathrm{rd}$ meetings of EWRC. Committee of methods in weed research. Weed Res., v. 4, n. 1, p. 88, 1964.

JAREMTCHUK, C. C., et al. Efeito de sistemas de manejo sobre a velocidade de dessecação, infestação inicial de plantas daninhas, desenvolvimento e produtividade de soja. Acta Sci. Agron., v. 30, n. 4, p. 449-455, 2008

LAND, P.; FRASCAROLI, E.; GIULIANI, M. M. Genetic variability for resistence to trifluralin in Zea mays. Weed Sci., v. 47 , n. 4 , p. $369-374,1999$

LORENZI, J. O.; PERESSIN, V. A. A cultura da mandioca Campinas: Instituto Agronômico de Campinas, 1989. 13 p.

LORENZI, J. O.; DIAS, C. A. C. Cultura da mandioca. Campinas: Coordenadoria de Assistência Técnica Integral CATI, 1993. 41 p. (Boletim Técnico, 211).
MELHORANÇA, A. L. Seletividade dos herbicidas diclosulam, flumetsulam e cloransulam em diversas cultivares de soja. In: REUNIÃO DE PESQUISADORES EM CONTROLE DE PLANTAS DANINHAS NOS CERRADOS, 12., 1999, Corumbá. Atas e Anais... Dourados Embrapa/CPAO, 1999. p. 15-18.

MESCHEDE, D. K. et al. Período anterior á interferência de plantas daninhas em soja: estudo de caso com baixo estande e testemunhas duplas. Planta Daninha, v. 22, n. 2, p. 239-246, 2004.

OLIVEIRA JR., R. S. et al. Manejo químico de plantas daninhas em áreas de plantio direto de mandioca. R. Bras. Herbic., v. 2, n. 3, p. 99-106, 2001a.

OLIVEIRA JR., R. S. et al. Tolerância de cinco cultivares de mandioca a diferentes herbicidas. Planta Daninha, v. 19, n. 1, p. $119-125,2001 b$.

PERESSIN, V. A. et al. Acúmulo de matéria seca na presença e na ausência de plantas infestantes no cultivar de mandioca SRT59 - Branca de Santa Catarina. Bragantia, v. 57, n. 1, p. 135-148, 1998.

PEREIRA, F. A. R. et al. Estudo da seletividade de herbicidas em cultivares de milho (Zea mays L.), nas condições ambientais de Mato Grosso do Sul. Ensaios Ci., v. 5, n. 2, p. 119-140, 2001.

SANTOS, M. J. C.; PAIVA, S. N. Os sistemas agroflorestais como alternativa econômica em pequenas propriedades rurais: estudo de caso. Ci. Flor., v. 12, n. 1, p. 135-141, 2002.

VELINI, E. et al. Avaliação dos efeitos do herbicida clomazone, aplicado em pós-emergência inicial, sobre o crescimento e produtividade de soqueira de cana-de-açúcar (Saccharum officinarum cv. SP 71-1406). STAB Açúcar, Álcool Subpr., v. 10, p. 13-16, 1992.

WILSON, R. G. Response of nine sugarbeet (Beta vulgaris) cultivars to postemergence herbicide applications.

Weed Technol., v. 13, n. 1, p. 25-29, 1999.

WYCHEN, L. R. V. et al. Tolerance of sweet corn (Zea mays) hybrids to RPA 201772. Weed Technol., v. 13, n. 2 , p. 221-226, 1999. 